\title{
Brucellosis relapse causing thoracic aortic ulcers and aneurysm formation: a case report
}

Shuai Li and Qiang Wang

\begin{abstract}
Background: Brucellosis is an infectious disease caused by Brucella spp, which can involve the cardiovascular, digestive, and respiratory systems. Cardiovascular involvement is a rare occurrence, it has an extremely high mortality rate.

Case presentation: A 67-year-old Chinese man presented with thoracic aortic multiple ulcers and partial aneurysm formation that caused symptoms of left waist and left buttock pain. The man was admitted to our hospital due to abdominal aorta pseudoaneurysms 5 years ago. The diagnosis was made by thoracic computed tomography angiography (CTA), previous history, and positive culture of Brucella, and the patient was successfully treated by thoracic aortic covered stent-graft implantation and specific medical treatment.

Conclusions: People who have a history of contact with cattle and sheep, should beware of the possibility of Brucella infection. If chest and abdominal pain occur, timely medical treatment is recommended, aortic aneurysm, the disease with a high risk of death, can be identified or excluded by CTA. Early treatment and prevention of disease progression are more beneficial to patients.
\end{abstract}

Keywords: Brucellosis, Relapse, Thoracic aortic ulcer, Aneurysm

\section{Background}

Brucella belongs to Gram-negative bacteria, often parasitizes in sheep, cattle, and other animals. In China, it is most common that infecting and causing disease through sheep. Brucellosis is a common zoonosis that is prevalent worldwide and can affect various systems throughout the body. We reported a case of previously treated brucellosis combined with abdominal aortic pseudoaneurysms. He was present thoracic aortic multiple ulcers and partial aneurysm formation due to brucellosis relapse. We performed thoracic aortic stent implantation under general anesthesia. Such cases have not been reported.

*Correspondence: qiangwang97@aliyun.com

Department of Infectious Disease, Shanxi Provincial People's Hospital, Taiyuan, China

\section{Case presentation}

A 67-year-old Chinese man, with long-standing high blood pressure and type 2 diabetes mellitus, presented with left waist and left buttock pain for 1 week. Blood cultures grew Brucella, and the CTA revealed multiple thoracic aortic ulcers and partial aneurysm formation. He was diagnosed with brucellosis. Five years ago, he suffered from an abdominal aorta pseudoaneurysm, and underwent surgery. During hospitalization, blood culture grew Brucella. After departure from the hospital, he was treated with a combination of rifampicin and doxycycline, but blood cultures grew Brucella many times. The patient described that he worked in a restaurant in Inner Mongolia five years ago, but he has no contact history with cattle or sheep since he was discharged from hospital. After this admission, the patient's body temperature, blood pressure, heart rate and respiration were normal, and no other abnormalities were found in physical examination. Laboratory studies revealed the white blood cell count $(\mathrm{WBC})$ was $5.15 \times 10^{9} / \mathrm{L}$, the hemoglobin $(\mathrm{Hb})$ was 
$122 \mathrm{~g} / \mathrm{L}$, the platelet count (PLT) was $152 \times 10^{9} / \mathrm{L}$, the C-reactive protein (CRP) was $17.28 \mathrm{mg} / \mathrm{L}$, the erythrocyte sedimentation rate (ESR) was $20 \mathrm{~mm} / \mathrm{h}$, the triglyceride was $2.33 \mathrm{~mol} / \mathrm{L}$, the low-density lipoprotein was $3.42 \mathrm{~mol} / \mathrm{L}$, normal electrolyte and coagulation indexes, normal electrocardiogram (ECG). The blood creatinine was $125.02 \mu \mathrm{mol} / \mathrm{L}$. The renal artery imaging showed that the blood perfusion of both kidneys was fair, the uptake and removal of imaging agents in the cortical cortex of both kidneys were reduced, the function was mild to moderate impaired, and the upper urinary drainage of both kidneys was fair. The glomerular filtration rate was $54.1 \mathrm{~mL} / \mathrm{min}$ (normal $>73.0 \mathrm{~mL} / \mathrm{min}$ ), $30.7 \mathrm{ml} / \mathrm{min}$ on the left side and $23.4 \mathrm{~mL} / \mathrm{min}$ on the right side. Explain to the patient and family, extensive use of contrast agents may worsen kidney injury during the operation, but the patient may at any time for thoracic aortic ulcer aggravating life-threatening, or intimal plaque off cause distal arterial embolism, the patient and family said they understood the disease and the risks and agreed to the operation, we performed thoracic aortic stent implantation under general anesthesia. Seen through imaging by catheter angiography: the diameter of the distal aortic arch of the left common carotid artery was about $2.9 \mathrm{~cm}$, the nearest ulcer was located on the opposite side of the left subclavian artery and the minor curvature of the aortic arch, the farthest thoracic aortic ulcer was located in the thoracic 9-10 intervertebral space. A 32-160 thoracic aortic stent (ANKURA, XJZDZ32160) was placed along the guide-wire at the level of the ascending aorta. The beginning of the coated end was located at the distal opening of the left common carotid artery. A 32-26200 aortic stent (ANKURA, TAA3226B200) was placed along the guide-wire, and the distal position of the stent was about $2.5 \mathrm{~cm}$ beyond the ulcer at the lowest end of the thoracic aorta. A 9-39 mm stent (Micro Medical, ICLC9039L) was placed along the guide-wire with the proximal end beyond the coated skin frame of the thoracic aorta and the distal end within the left subclavian artery. After then, catheter angiography showed: ascending aorta, aortic arch, truncus brachiocephalicus, left common carotid artery, left subclavian artery blood flow were smooth, and the blood vessel wall is smooth. The operation was successful. The patient's vital signs remained stable throughout the operation. The patients were followed up 6 weeks after the surgery and had no complaints of any special discomfort.

\section{Discussion and conclusions}

Brucellosis is a zoonotic infectious disease caused by Brucella spp. It is often caused by the ingestion of be contaminated undercooked meat and dairy products, and the contact history of related animals such as sheep and cattle [1], so the risk of the disease is higher in related occupations such as keepers and dairy workers. Brucella can cause multisystem involvement, but cardiovascular involvement is less common. Endocarditis, which occurs in 1-2\% of cases [2], has a high risk of death, accounting for more than $75 \%$ of the deaths of brucellosis [3, 4]. Brucella is an intracellular parasitic bacterium. Bacteria and toxins play a major role in the acute phase, while delayed allergic reaction and the formation of granuloma are the main ones in the chronic phase [5]. Although Brucella can lead to endovasculitis if it affects blood vessels [5], the vascular complications of brucellosis are rare. In a review of 25 cases of brucellosis with aortic involvement, Kakkos et al. found that $65 \%$ of the abdominal aorta and $23 \%$ of ascending aorta were involved [6]. Only a small number of cases have been reported in the past 5 years [7-12], and only one case has been reported recurrent in the past 10 years, presenting as prosthetic valve endocarditis and aortic root infective pseudoaneurysms[13]. The aortic involvement typically showing the formation of major aneurysm or large aneurysm. Brucellosis causes severe inflammatory reaction by infection of abnormal vascular tissues, such as intimal defect caused by atherosclerosis [14], activating endothelial cells, up-regulating adhesion molecules, and secreting pro-inflammatory chemokines [15], leading to severe inflammatory reactions. As a result of Brucella infection, artery endothelium is damaged, and the middle layer is exposed to the blood flow, then forming arterial ulcers, aortic dissection, aortic aneurysm and aortic rupture.

At admission, the patient had left waist and left buttock pain for 1 week. Review with five years ago, the patient had visited our hospital due to Brucella abdominal aortic pseudoaneurysm. The symptoms were left upper abdominal pain for 1 month, transferred to the back pain for 20 days. He had a contact history of cattle, but no contact history of cattle has been found since his departure from the hospital. Therefore, the patient had similar symptoms, CTA examination showed multiple ulcers of thoracic aorta and the formation of partial aneurysm, which was most likely caused by the relapse of previously acquired infection, but the symptoms may be atypical due to the short course of disease.

In conclusion, as a case of multiple thoracic aortic ulcers and partial aneurysm formation due to brucellosis relapse, and the patient was successfully treated by thoracic aortic covered stent-graft implantation and specific medical treatment. However, this case still has some limitations: (1) Intraoperative pathological analysis was not obtained, and the diagnosis was based on CTA, previous history and blood culture results; (2) The postoperative follow-up was only 6 weeks, we will further follow-up. However, people who have a history of contact 
with cattle and sheep, should beware of the possibility of Brucella infection. Rose bengal plate test (RBPT) is feasible to conduct preliminary screening. During treatment follow-up, non-specific tests such as ESR and CRP should also be used besides for serological tests [16]. If chest and abdominal pain occur, timely medical treatment is recommended, aortic aneurysm, the disease with a high risk of death, can be identified or excluded by CTA. Early treatment and prevention of disease progression are more beneficial to patients (Fig. 1).

\section{Abbreviations}

CTA: Computed tomography angiography; WBC: White blood cell count; $\mathrm{Hb}$ : Hemoglobin; PLT: Platelet; CRP: C-reactive protein; ESR: Erythrocyte sedimentation rate; ECG: Electrocardiogram; RBPT: Rose Bengal plate test.

\section{Acknowledgements}

Not applicable.

\section{Authors' contributions}

LS: was responsible for material collecting and manuscript drafting. LS, WQ: contributed to interpreting the clinical materials, designing the structure of manuscript, revising and modifying the manuscript critically focusing on

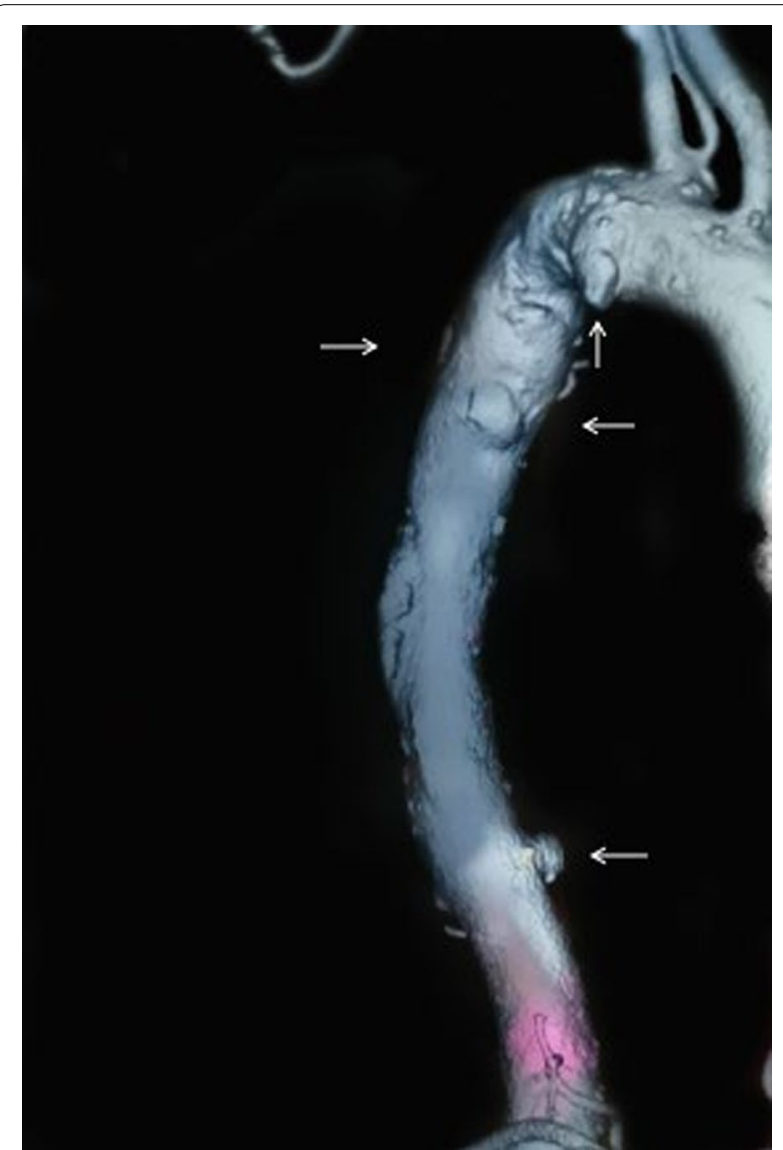

Fig. 1 Computed tomography angiography (CTA): Multiple penetrating ulcers in thoracic aorta, partial aneurysm formation important intellectual content. WQ: provided important intellectual contents, reviewed the literature and offered valuable suggestions to improve the manuscript. All authors gave approval to the final version which would be submitted. Both authors read and approved the final manuscript.

\section{Funding}

Not applicable.

\section{Availability of data and materials}

The datasets used during the current study are available from the first author upon reasonable request.

\section{Declarations}

Ethics approval and consent to participate

Not applicable.

\section{Consent for publication}

Written informed consent was obtained from the patient for publication of this case report and any accompanying images.

\section{Competing interests}

The authors declare that they have no competing interests.

Received: 29 April 2021 Accepted: 22 December 2021

Published online: 10 January 2022

\section{References}

1. Colmenero JD, Reguera JM, Martos F, Sánchez-De-Mora D, Delgado M, Causse M, Martín-Farfán A, C J. Complications associated with Brucella melitensis infection: a study of 530 cases. Medicine. 1996;75(4):195-211.

2. Cakalagaoglu C, Keser N. Brucella-mediated prosthetic valve endocarditis with brachial artery mycotic aneurysm. J Heart Valve Dis. 1999:8(5):586-90.

3. Reguera JM, Alarcón A, Miralles F, Pachón J, Juárez C. Brucella endocarditis: clinical, diagnostic, and therapeutic approach. Eur J Clin Microbiol Infect Dis. 2003;22(11):647-50

4. Pappas G, Akritidis N, Bosilkovski M. Brucellosis. Engl J med. 2005;352(22):2325-36.

5. Wen-hong Z, Zhang Y-X. Brucella diagnosis and treatment expert consensus. Chin J Infect Dis. 2017;35(12):705-10.

6. Kakkos SK, Papadoulas S, Lampropoulos G, Marangos M, Kalogeropoulou C, IA T. Aorto-iliac aneurysm infected by Brucella: distinctive presentation patterns of a rare entity. Vascular. 2013;21(5):307-15.

7. Wang S, Wang Q, Liu H, Sun S, Sun X, Zhang Y, Wang Z. Endovascular treatment of thoracic aortic pseudoaneurysm due to brucellosis: a rare case report. BMC Infect Dis. 2017;17(1):387.

8. Alsheef M, Alsaleh S, Alanezi N, Bakhsh N, AlDosary R, AlSharif L, Mian A, Ahsan A. Descending thoracic aortic aneurysm due to brucella melitensis. Hindawi. 2019;2019:4939452

9. Zhang T, Ji D. Endovascular treatment of Brucella-infected abdominal aortic aneurysm: A case report. Medicine. 2017;96(42):e7666.

10. Alhaizaey A, Alassiri M, Alghamdi M. Mycotic aortic aneurysm due to brucellosis. J Vasc Surg Cases Innov Tech. 2016;2(2):50-2.

11. Fuchs I, Taylor J, Malev A. Definitive endovascular repair of a brucellar descending thoracic aortic aneurysm. Isr Med Assoc J. 2017;19(5):325-7.

12. Li H-L, Chan YC, Cui D-Z, Li N, Cheng SW. Successful endovascular aneurysm repair for brucella mycotic aneurysm acquired from ingestion of sheep placenta. Ann Vasc Surg. 2019;57:274-274.

13. Amirghofran AA, Karimi A, Emaminia A, Sharifkazemi MB. Brucellosis relapse causing prosthetic valve endocarditis and aortic root infective pseudoaneurysm. Ann Thorac Surg. 2011;92(4):e77-9.

14. Cascio A, De Caridi G, Lentini S, Benedetto F, Stilo F, Passari G, laria C, Spinelli F. Involvement of the aorta in brucellosis: the forgotten, lifethreatening complication. A systematic review. Vector Borne Zoonotic Dis. 2012;12(10):827-40 
15. Ferrero MC, Bregante J, Delpino MV, Barrionuevo P, Fossati CA, Giambartolomei GH, PC B. Proinflammatory response of human endothelial cells to Brucella infection. Microbes Infect. 2011;13(10):852-61.

16. Buzgan T, Karahocagil MK, Irmak H, Baran Al, Karsen H, Evirgen O, Akdeniz H. Clinical manifestations and complications in 1028 cases of brucellosis: a retrospective evaluation and review of the literature. Int $J$ Infect Dis. 2010;14(6):e469-78.

\section{Publisher's Note}

Springer Nature remains neutral with regard to jurisdictional claims in published maps and institutional affiliations.

- fast, convenient online submission

- thorough peer review by experienced researchers in your field

- rapid publication on acceptance

- support for research data, including large and complex data types

- gold Open Access which fosters wider collaboration and increased citations

- maximum visibility for your research: over $100 \mathrm{M}$ website views per year

At BMC, research is always in progress.

Learn more biomedcentral.com/submissions 